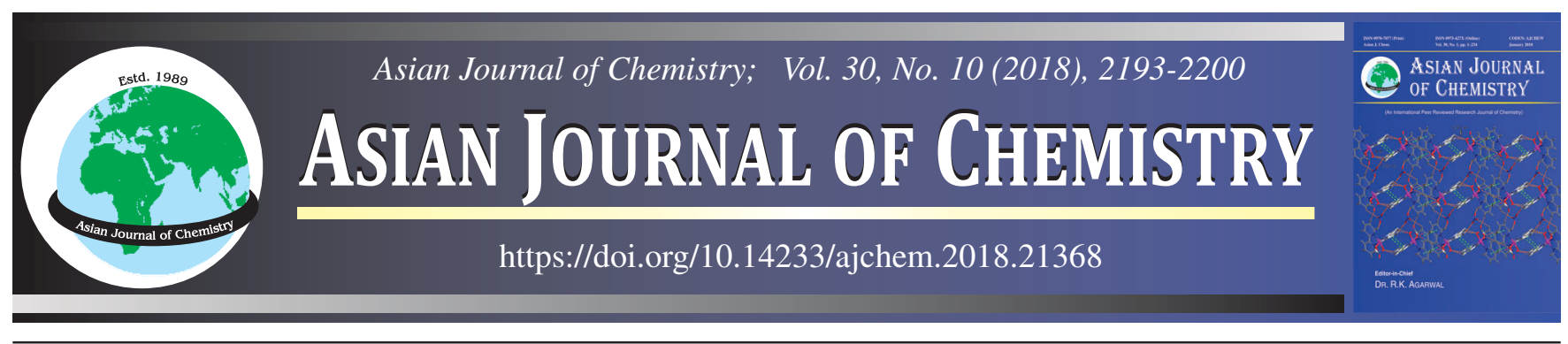

\title{
Design and Synthesis of Novel $N$-(4-(Pyridin-2-yloxy)benzylidene)-4- [4-(substituted)phenyl]semicarbazides as Potential Anticonvulsant Agents
}

\author{
Prem Singh $^{1}$ and LaXmi Tripathi ${ }^{2, *}$
}

${ }^{1}$ IFTM University, Moradabad-244 001, India

${ }^{2}$ Moradabad Educational Trust, Group of Institutions, Faculty of Pharmacy, Moradabad-244 001, India

*Corresponding author: E-mail: tripathilaxmi1979@gmail.com

Received: 6 April 2018;

Accepted: 23 July 2018;

Published online: 31 August 2018;

AJC-19042

A new series of $N$-(4-(pyridin-2-yloxy)benzylidene)-4-[4-(substituted)phenyl]semicarbazides (PSSD1-8) were designed and synthesized
keeping in view the structural requirement of pharmacophore and evaluated for their possible anticonvulsant activity. All the derivatives
were synthesized by the given scheme and reaction process was monitored by thin layer chromatography. The structure of synthesized
derivatives was confirmed by FT-IR, ${ }^{1} \mathrm{H}$ NMR, mass spectroscopy and elemental analysis. The anticonvulsant activity was established
after intraperitoneal administration in MES and scMET seizure models. The most active compound of the series was $1-(4-($ pyridin-2-
yloxy)-benzylidene)-4- $p$-tolylsemicarbazide (PSSD5). A molecular docking study was carried out in order to assess the interaction and |
binding modes with target receptor/enzyme. Titled compounds were found to strongly bind to human gamma-aminobutyric acid receptor
(GABA ${ }_{\mathrm{A}} \mathrm{R}-\beta_{3}$ ). A computational study was also carried to predict the pharmacokinetic properties of the synthesized compounds.
Keywords: Semicarbazone, Anticonvulsant Activity, Neurotoxicity, Molecular docking, Computational study.

\section{INTRODUCTION}

Anticonvulsants constitute a varied group of drugs used for treating epileptic seizures. The objective of administering anticonvulsants is to suppress the rapid and uncontrolled activation of neurons that start a seizure. The non-selectivity and adverse side effects caused by the use of currently available antiepileptic drugs have propelled the search for antiepileptic compounds with selective activity and relatively low toxicity [1]. A survey of literature revealed that the semicarbazones have emerged as compounds with a myriad of biological activities including anticonvulsant, anticancer, antitubercular and antimicrobial activities [2]. Semicarbazones are currently being used to develop versatile anticonvulsant pharmacophores. These compounds were reported to exhibit potent anticonvulsant effects in various preclinical anticonvulsant models. Thus far, various semicarbazone derivatives containing 1,3,4thiadiazole,1,3,4-oxadiazole, pyrimidine, benzothiazole and substituted phenyl or aryl rings have been synthesized. The anticonvulsant activities of these derivatives have been evaluated. Anticonvulsant activity is only observed when a semicarbazone based pharmacophore model consisting of four binding sites is used. The model consists of an aryl hydrophobic binding site, hydrogen-bonding domain, an electron donating group and another hydrophobic-hydrophilic site regulating the pharmacokinetic properties of the anticonvulsant [3].

In this study, we reported the synthesis and anticonvulsant activities of $\mathrm{N}$-(4-(pyridin-2-yloxy)benzylidene)-4-[4-(substituted)phenyl]semicarbazides. FT-IR, MS, ${ }^{1} \mathrm{H}$ NMR and elemental analysis were used for the characterization of the synthesized semicarbazides. All the synthesized compounds consisted of the pharmacophoric elements (Fig. 1) that are essential for acceptable anticonvulsant activity, namely a hydrophobic binding site (A), a hydrogen-bonding domain (HBD), a hydrophobichydrophilic site (C) and an electron-donating group (D) [4]. The anticonvulsant activities of semicarbazides were experimentally evaluated using the maximal electroshock and subcutaneous metrazole tests in epilepsy mouse models. The neurotoxicity of the semicarbazides in mice was evaluated through the rotarod assay. The molecular docking studies of designed molecules were performed to assess the interaction and modes of binding of the synthesized compounds with target receptor/

This is an open access journal, and articles are distributed under the terms of the Creative Commons Attribution-NonCommercial 4.0 International (CC BY-NC 4.0) License, which allows others to copy and redistribute the material in any medium or format, remix, transform, and build upon the material, as long as appropriate credit is given and the new creations are licensed under the identical terms. 


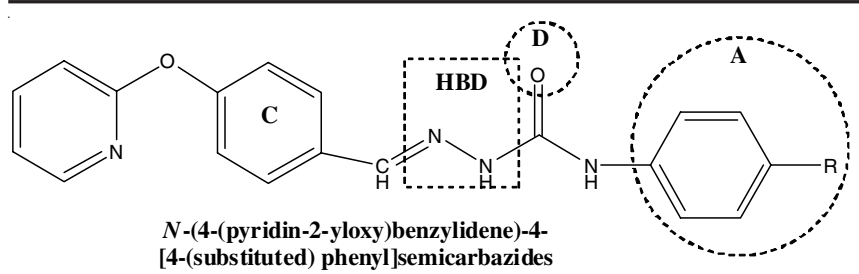

Fig. 1. Essential pharmacophoric elements for anticonvulsant activity

enzyme using the Glide extra precision (XP) Maestro 10.1 Schrodinger software. A computational study was also performed to predict the pharmacokinetic parameters and Log P values.

\section{EXPERIMENTAL}

All the chemicals were purchased from Sigma and Himedia Chemicals, supplied by S.K. Traders Indore, India. Melting points were determined on a Tempo capillary melting point apparatus, Mumbai, India and are uncorrected. The FT-IR spectrum was recorded $\left(\lambda_{\max }\right.$ in $\left.\mathrm{cm}^{-1}\right)$ on Bruker Tensor $27 \mathrm{FT}$ IR spectrometer. ${ }^{1} \mathrm{H}$ NMR spectrum were recorded at $300 \mathrm{MHz}$, after dissolving in a suitable solvent (DMSO or $\mathrm{D}_{2} \mathrm{O}$ ) on Bruker Avance II $400 \mathrm{MHz}$, USA FT-NMR spectrometer using tetramethylsilane as internal standard and chemical shifts $(\delta)$ are reported in ppm. The purity of the compounds was ascertained by thin layer chromatography (TLC) and elemental analysis. Plates for TLC were prepared with silica gel $\mathrm{G}$ and activated at $110^{\circ} \mathrm{C}$ for $30 \mathrm{~min}$. Iodine vapours was used to develop the TLC plates. The mass spectra were recorded on a Waters Micromass ZQ 2000 mass spectrometer. Elemental analyses were performed on a Vario EL-III analyzer.

Synthesis of 4-(pyridin-2-yloxy)benzaldehyde (3): A mixture of $o$-chloropyridine (1) $(0.002 \mathrm{~mol}), p$-hyroxybenzaldehyde (2) (0.002 mol), potassium carbonate $(0.002 \mathrm{~mol})$ and $10 \mathrm{~mL}$ of dry dimethyl formamide was taken in a round bottom flask and heated at $70{ }^{\circ} \mathrm{C}$ for 3-4 h under reduced pressure. Reaction mixture was poured into water and extracted with ethyl acetate. The combined extracts were washed with water and dried over anhydrous sodium sulfate. Yield: $66.8 \%$; m.w. 199.21; m.p.: $79-81{ }^{\circ} \mathrm{C}$. The purity of compound was checked by TLC using silica gel G; solvent system ethyl acetate:ethanol (3:2) and detecting agent: iodine vapours. Only one sport was obtained $\left(\mathrm{R}_{\mathrm{f}} 0.65\right)$ [5].

Synthesis of 4-(substituted)phenyl urea (5): Aniline and/ or $p$-substituted aniline (4) $(0.1 \mathrm{~mol})$ was dissolved in glacial acetic acid $(10 \mathrm{~mL})$ and diluted with water (upto $100 \mathrm{~mL}$ ). To this solution an equimolar $(0.1 \mathrm{~mol})$ quantity of sodium cyanate in warm water $(50 \mathrm{~mL})$ was added with stirring. The reaction mixture was allowed to stand for $30 \mathrm{~min}$, and then compounds were filtered, washed with water and dried after recrystallization from boiling water [6].

Synthesis of 4-(substituted)phenyl semicarbazides (6): To an aqueous solution of 4-(substituted)phenyl urea (5) (0.1 $\mathrm{mol})$, an equimolar quantity of hydrazine hydrate and ethanol $(2 \mathrm{~mL}$ ) was added. The reaction mixture was refluxed for $30 \mathrm{~min}$ and cooled in ice. Sodium hydroxide ( $4 \mathrm{~g}$ ) was added to make the reaction mixture alkaline, before refluxing. The product was filtered under suction and recrystallized from ethanol [6].

Synthesis of $N$ - (4-(pyridin-2-yloxy)benzylidene)-4-[4(substituted)phenyl]semicarbazide (PSSD1-8) : A mixture of 4-(pyridin-2-yloxy)benzaldehyde (3) (0.002 mol), 4-(substituted) -phenylsemicarbazides (6) $(0.002 \mathrm{~mol})$ and $10 \mathrm{~mL}$ of ethanol were taken in a round bottom flask. Few drops of glacial acetic acid was added and refluxed for $2-3 \mathrm{~h}$. The reaction was monitored by TLC and the reaction mixture was poured into ice. The precipitate was filtered and washed with sodium acetate $(0.005 \mathrm{~mol})$. The crude solid was dried and recrystallized with hot ethanol to obtain compounds (PSSD1-PSSD8) (Scheme-I) [7].

$N$-(4-(pyridin-2-yloxy)benzylidene)-4-phenylsemicarbazide (PSSD1): Yield: $62.15 \%$, m.p. $168-170{ }^{\circ} \mathrm{C}$, m.f. $\mathrm{C}_{19} \mathrm{H}_{16} \mathrm{~N}_{4} \mathrm{O}_{2}$; m.w. 332.36, $\mathrm{R}_{\mathrm{f}}$ 0.53. FT-IR (KBr, $\left.v_{\max }, \mathrm{cm}^{-1}\right)$ : 3429, 3315 (N-H str.), 3030 (C-H str.), 2875 (=C-H str.), 20001670 (over-tone for mono-substitution on aromatic ring), 1659 (C=O str.), 1637 (azomethine $\mathrm{C}=\mathrm{N}$ str.), 1597 (phenyl ring str.), 1465 (phenyl C-H in plane bending), 1282 (C-N str. coupled with N-H bending), 1252 (C-O str.), 1042 (N-N str.), 785 \& 708 (C-H out of plane bending for mono-substitution). ${ }^{1} \mathrm{H}$ NMR $\left(\mathrm{CDCl}_{3}, 300 \mathrm{MHz}\right) \delta$ in ppm: $8.35\left(\mathrm{~s}, 1 \mathrm{H}, \mathrm{Ph}-\mathrm{C} \underline{\mathrm{H}}=\mathrm{N}\right.$ at $\left.\mathrm{N}_{1}\right), 7.78-$ $7.76\left(\mathrm{~d}, 2 \mathrm{H}, o\right.$-benzyl ring protons at $\left.\mathrm{N}_{1}\right), 7.54-7.42(\mathrm{~d}, 1 \mathrm{H}$, pyridine ring proton at $\left.\mathrm{C}_{6}\right), 7.32-7.30(\mathrm{~d}, 2 \mathrm{H}, o$-phenyl ring protons at $\left.\mathrm{N}_{4}\right), 7.22-7.17\left(\mathrm{t}, 3 \mathrm{H}, m\right.$ - \& $p$-phenyl ring protons at $\mathrm{N}_{4}$ ), 6.94-6.80 ( $\mathrm{m}, 3 \mathrm{H}$, pyridine ring proton at C3 \& C5), 6.66$6.58\left(\mathrm{~d}, 2 \mathrm{H}, m\right.$-benzyl ring protons at $\left.\mathrm{N}_{1}\right), 6.23(\mathrm{~s}, 1 \mathrm{H},-\mathrm{C}=\mathrm{N}-$ N $\underline{\mathrm{H}}$ semicarbazide proton), 6.21 (s, $1 \mathrm{H}, \mathrm{O}=\mathrm{C}-\mathrm{NH}$ semicarbazide proton). $\mathrm{MS}(\mathrm{m} / \mathrm{z}, \%): \mathbf{M}^{+}: 332.13,\left(\mathbf{M}^{+}+1: 333.18\right), \mathbf{M}^{+}-\mathrm{C}_{6} \mathrm{H}_{6}$ : 252.50, $\mathrm{M}^{+}-\mathrm{C}_{7} \mathrm{H}_{8} \mathrm{~N}_{2} \mathrm{O}: 204.20, \mathrm{M}^{+}-\mathrm{C}_{5} \mathrm{H}_{5} \mathrm{~N}: 257.11$. Anal. Calcd. (found) (\%): C, 68.66 (68.69); H, 4.85 (4.83); N, 16.86 (16.83); $\mathrm{O}, 9.63$ (9.67).

N-(4-(Pyridin-2-yloxy)benzylidene)-4-(4-chlorophenyl)semicarbazide (PSSD2): Yield: $68.94 \%$, m.p. $163-165{ }^{\circ} \mathrm{C}$, m.f. $\mathrm{C}_{19} \mathrm{H}_{15} \mathrm{~N}_{4} \mathrm{O}_{2} \mathrm{Cl}$; m.w. 366.80, $\mathrm{R}_{\mathrm{f}}$ 0.58. FT-IR (KBr, $v_{\max }$, $\mathrm{cm}^{-1}$ ): 3428, 3317 ( N-H str.), 3032 (C-H str.), 2877 (=C-H str.), 2000-1670 (overtone for disubstitution on aromatic ring), 1659 (C=O str.), 1639 (azomethine $\mathrm{C}=\mathrm{N}$ str.), 1590 (phenyl ring str.), 1467 (phenyl C-H in plane bending), 1282 (C-N str. coupled with N-H bending), 1252 (C-O str.), 738 (C-Cl str.), 1042 (N-N str.), 846 (C-H out of plane bending for disubstitution). ${ }^{1} \mathrm{H}$ NMR $\left(\mathrm{CDCl}_{3}, 300 \mathrm{MHz}\right) \delta$ in ppm: $8.59(\mathrm{~s}, 1 \mathrm{H}, \mathrm{Ph}-\mathrm{C} \underline{\mathrm{H}}=\mathrm{N}), 7.94-7.92$ $\left(\mathrm{d}, 2 \mathrm{H}, o\right.$-benzyl ring protons at $\left.\mathrm{N}_{1}\right), 7.90-7.88(\mathrm{~d}, 2 \mathrm{H}, o$-phenyl ring protons at $\left.\mathrm{N}_{4}\right), 7.70-7.62(\mathrm{t}, 1 \mathrm{H}$, pyridine ring proton at $\mathrm{C}_{4}$ ), 7.46-7.44 (d, 2H, $m$-phenyl ring protons at $\mathrm{N}_{4}$ ), 7.24-7.20 $\left(\mathrm{d}, 1 \mathrm{H}\right.$, pyridine ring proton at $\left.\mathrm{C}_{6}\right), 7.04-7.02(\mathrm{~d}, 2 \mathrm{H}, m$-benzyl ring protons at $\left.\mathrm{N}_{1}\right), 6.60-6.58(\mathrm{~d}, 1 \mathrm{H}$, pyridine ring proton at $\left.\mathrm{C}_{3}\right), 6.39-6.33\left(\mathrm{t}, 1 \mathrm{H}\right.$, pyridine ring proton at $\left.\mathrm{C}_{5}\right), 6.04(\mathrm{~s}, 2 \mathrm{H}$, semicarbazide $\mathrm{NH}$ protons). $\mathrm{MS}(\mathrm{m} / \mathrm{z}, \%): \mathrm{M}^{+}: 366.09,\left(\mathrm{M}^{+}+1\right.$ for ${ }^{35} \mathrm{Cl}: 367.18, \mathrm{M}^{+}+2$ for ${ }^{37} \mathrm{Cl}: 368.09$ ), $\mathrm{M}^{+}-\mathrm{C}_{6} \mathrm{H}_{5} \mathrm{Cl}: 257.16$, $\mathrm{M}^{+}-\mathrm{C}_{7} \mathrm{H}_{7} \mathrm{ClN}_{2} \mathrm{O}: 198.08, \mathrm{M}^{+}-\mathrm{C}_{5} \mathrm{H}_{5} \mathrm{~N}: 291.66$. Anal. Calcd. (found) (\%): C, 62.21 (62.24); H, 4.12 (4.09); Cl, 9.67 (9.65); N, 15.27 (15.25); O, 8.72 (8.76).

$\mathrm{N}$-(4-(Pyridin-2-yloxy)benzylidene)-4-(4-bromophenyl)semicarbazide (PSSD3): Yield: $67.51 \%$, m.p. 190-192 ${ }^{\circ} \mathrm{C}$, m.f. $\mathrm{C}_{19} \mathrm{H}_{15} \mathrm{~N}_{4} \mathrm{O}_{2} \mathrm{Br}$; m.w. 411.25, $\mathrm{R}_{\mathrm{f}}$ 0.52. FT-IR $\left(\mathrm{KBr}, \mathrm{v}_{\max }, \mathrm{cm}^{-1}\right)$ : 3442, 3326 (N-H str.), 3018 (C-H str.), 2917 ( =C-H str.), 20001670 (overtone for disubstitution on aromatic ring), $1665(\mathrm{C}=\mathrm{O}$ str.), 1645 (azomethine $\mathrm{C}=\mathrm{N}$ str.), 1586 (phenyl ring str.), 1462 (phenyl C-H in plane bending), 1276 (C-N str. coupled with $\mathrm{N}-\mathrm{H}$ bending), 1248 (C-O str.), 588 (C-Br str.), 1046 (N-N str.), 841 (C-H out of plane bending for disubstitution). ${ }^{1} \mathrm{H} \mathrm{NMR}\left(\mathrm{CDCl}_{3}\right.$, 
<smiles>Clc1ccccn1</smiles>

(1)<smiles>CCCCCCCCCC=O</smiles>

(3)<smiles>O=Cc1ccc(O)cc1</smiles>

(2)

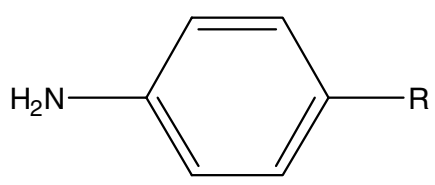

(4)

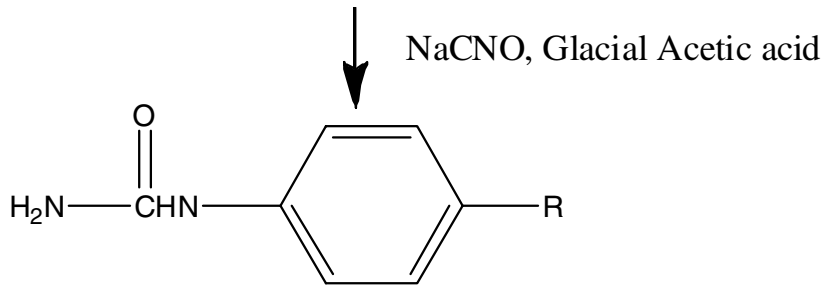

(5)<smiles>CC(C)NN</smiles><smiles>[R]c1ccc(NC(N)=O)cc1</smiles>

(6)<smiles>[R]c1ccc(NC(=O)N/N=C/c2ccc(Oc3ccccn3)cc2)cc1</smiles>

\section{N-(4-(pyridin-2-yloxy)benzylidene)-4-[4-(substituted)phenyl]semicarbazide}

Scheme-I: Synthetic scheme for synthesis of $N$-(4-(pyridin-2-yloxy)benzylidene)-4-[4-(substituted) phenyl]semicarbazide (PSSD1-PSSD8)

$300 \mathrm{MHz}) \delta$ in ppm: $8.62(\mathrm{~s}, 1 \mathrm{H}, \mathrm{Ph}-\mathrm{C} \underline{\mathrm{H}}=\mathrm{N}), 7.92-7.90(\mathrm{~d}$, $2 \mathrm{H}, o$-benzyl ring protons at $\left.\mathrm{N}_{1}\right), 7.86-7.82(\mathrm{~d}, 2 \mathrm{H}, o$-phenyl ring protons at $\left.\mathrm{N}_{4}\right), 7.72-7.64(\mathrm{t}, 1 \mathrm{H}$, pyridine ring proton at $\left.\mathrm{C}_{4}\right), 7.48-7.41\left(\mathrm{~d}, 2 \mathrm{H}, m\right.$-phenyl ring protons at $\left.\mathrm{N}_{4}\right), 7.26-7.22$ $\left(\mathrm{d}, 1 \mathrm{H}\right.$, pyridine ring proton at $\left.\mathrm{C}_{6}\right), 7.08-7.06(\mathrm{~d}, 2 \mathrm{H}, m$-benzyl ring protons at $\left.\mathrm{N}_{1}\right), 6.64-6.62(\mathrm{~d}, 1 \mathrm{H}$, pyridine ring proton at $\left.\mathrm{C}_{3}\right), 6.39-6.35\left(\mathrm{t}, 1 \mathrm{H}\right.$, pyridine ring proton at $\left.\mathrm{C}_{5}\right), 6.08(\mathrm{~s}, 2 \mathrm{H}$, semicarbazide $\mathrm{N} \underline{\mathrm{H}}$ protons). $\mathrm{MS}(\mathrm{m} / \mathrm{z}, \%)$ : $\mathbf{M}^{+}: 410.04,\left(\mathbf{M}^{+}+1\right.$ for ${ }^{79} \mathrm{Br}: 412.11, \mathrm{M}^{+}+2$ for $\left.{ }^{81} \mathrm{Br}: 411.06\right), \mathrm{M}^{+}-\mathrm{C}_{6} \mathrm{H}_{5} \mathrm{Br}: 257.10$, $\mathrm{M}^{+}-\mathrm{C}_{7} \mathrm{H}_{7} \mathrm{BrN}_{2} \mathrm{O}: 199.08, \mathrm{M}^{+}-\mathrm{C}_{5} \mathrm{H}_{5} \mathrm{~N}: 334.01$. Anal. Calcd. (found) (\%): C, 55.49 (55.56); H, 3.68 (3.72); Br, 19.43 (19.39); N, 13.62 (13.65); O, 7.78 (7.81).

$\mathrm{N}$-(4-(Pyridin-2-yloxy)benzylidene)-4-(4-fluorophenyl)semicarbazide (PSSD4): Yield: $62.14 \%$, m.p. $160-162{ }^{\circ} \mathrm{C}$, m.f. $\mathrm{C}_{19} \mathrm{H}_{15} \mathrm{~N}_{4} \mathrm{O}_{2}$ F; m.w. $350.35 \mathrm{~m}, \mathrm{R}_{\mathrm{f}}$ 0.62. FT-IR ( $\mathrm{KBr}, v_{\max }$, $\mathrm{cm}^{-1}$ ): 3434, 3322 (N-H str.), 3026 (C-H str.), 2897 (=C-H str.), 2000-1675 (overtone for disubstitution on aromatic ring), 1675 $(\mathrm{C}=\mathrm{O}$ str.), 1632 (azomethine $\mathrm{C}=\mathrm{N}$ str.), 1594 (phenyl ring str.), 1459 (phenyl C-H in plane bending), 1284 (C-N str. coupled with N-H bending), 1258 (C-O str.), 1138 (C-F str.), 1039 (N-N str.), 852 (C-H out of plane bending for disubstitution). ${ }^{1} \mathrm{H}$ NMR $\left(\mathrm{CDCl}_{3}, 300 \mathrm{MHz}\right) \delta$ in ppm: $8.43(\mathrm{~s}, 1 \mathrm{H}, \mathrm{Ph}-\mathrm{C} \underline{\mathrm{H}}=\mathrm{N}), 7.86-7.84$ $\left(\mathrm{d}, 2 \mathrm{H}, o\right.$-benzyl ring protons at $\left.\mathrm{N}_{1}\right), 7.80-7.78(\mathrm{~d}, 2 \mathrm{H}, o$-phenyl ring protons at $\left.\mathrm{N}_{4}\right), 7.74-7.64(\mathrm{t}, 1 \mathrm{H}$, pyridine ring proton at
$\left.\mathrm{C}_{4}\right)$, 7.54-7.52 (d, $2 \mathrm{H}, m$-phenyl ring protons at $\left.\mathrm{N}_{4}\right), 7.34-7.32$ $\left(\mathrm{d}, 1 \mathrm{H}\right.$, pyridine ring proton at $\left.\mathrm{C}_{6}\right), 7.11-7.08(\mathrm{~d}, 2 \mathrm{H}, m$-benzyl ring protons at $\left.\mathrm{N}_{1}\right), 6.60-6.58(\mathrm{~d}, 1 \mathrm{H}$, pyridine ring proton at $\left.\mathrm{C}_{3}\right), 6.52-6.48\left(\mathrm{t}, 1 \mathrm{H}\right.$, pyridine ring proton at $\left.\mathrm{C}_{5}\right), 6.05(\mathrm{~s}, 2 \mathrm{H}$, semicarbazide $\mathrm{N} \underline{\mathrm{H}}$ protons). $\mathrm{MS}(\mathrm{m} / \mathrm{z}, \%): \mathbf{M}^{+}: 350.12,\left(\mathbf{M}^{+}+1\right.$ for $\left.{ }^{19} \mathrm{~F}: 351.11\right), \mathrm{M}^{+}-\mathrm{C}_{6} \mathrm{H}_{5} \mathrm{~F}: 258.10, \mathrm{M}^{+}-\mathrm{C}_{7} \mathrm{H}_{7} \mathrm{~N}_{2} \mathrm{OF}: 199.08$, $\mathrm{M}^{+}-\mathrm{C}_{5} \mathrm{H}_{5} \mathrm{~N}$ : 274.09. Anal. Calcd. (found) (\%): C, 65.14 (65.18); H, 4.32 (4.28); N, 15.99 (15.88); O, 9.13 (9.11); F, 5.42 (5.46).

$\mathrm{N}$-(4-(Pyridin-2-yloxy)benzylidene)-4-p-tolylsemicarbazide (PSSD5): Yield: $78.86 \%$, m.p. $134-136{ }^{\circ} \mathrm{C}$, m.f. $\mathrm{C}_{20} \mathrm{H}_{18} \mathrm{~N}_{4} \mathrm{O}_{2}$; m.w. 346.38, $\mathrm{R}_{\mathrm{f}}$ 0.56. FT-IR (KBr, $v_{\max }, \mathrm{cm}^{-1}$ ): 3422, 3312 (N-H str.), 3028, 2967, 2876 (C-H str.), 2000-1670 (overtone for disubstitution on aromatic ring), $1657(\mathrm{C}=\mathrm{O}$ str.), 1642 (azomethine $\mathrm{C}=\mathrm{N}$ str.), 1598 (phenyl ring str.), 1464 (phenyl C-H in plane bending), 1450 (asym. methyl C-H bending), 1377 (sym. methyl C-H bending), 1280 (C-N str. coupled with N-H bending), 1250 (C-O str.), 1045 (N-N str.), 844 (C-H out of plane bending for disubstitution). ${ }^{1} \mathrm{H} \mathrm{NMR}\left(\mathrm{CDCl}_{3}, 300 \mathrm{MHz}\right) \delta$ in ppm: $8.38(\mathrm{~s}, 1 \mathrm{H}, \mathrm{Ph}-\mathrm{CH}=\mathrm{N}), 7.86-7.82(\mathrm{t}, 1 \mathrm{H}$, pyridine ring proton at $\left.\mathrm{C}_{4}\right), 7.72-7.70\left(\mathrm{~d}, 1 \mathrm{H}\right.$, pyridine ring proton at $\left.\mathrm{C}_{6}\right)$, 7.64-7.62 (d, 2H, $m$-phenyl ring protons at $\left.\mathrm{N}_{4}\right), 7.52-7.50(\mathrm{~d}$, $2 \mathrm{H}, o$-benzyl ring protons at $\left.\mathrm{N}_{1}\right), 7.14-7.12(\mathrm{~d}, 2 \mathrm{H}, m$-phenyl ring protons at $\left.\mathrm{N}_{4}\right), 7.98-7.96(\mathrm{~d}, 2 \mathrm{H}, m$-benzyl ring protons at $\left.\mathrm{N}_{1}\right), 6.74-6.72\left(\mathrm{~d}, 1 \mathrm{H}\right.$, pyridine ring proton at $\left.\mathrm{C}_{3}\right), 6.48-6.44$ 
$\left(\mathrm{t}, 1 \mathrm{H}\right.$, pyridine ring proton at $\left.\mathrm{C}_{5}\right), 6.11(\mathrm{~s}, 2 \mathrm{H}$, semicarbazide $\mathrm{N} \underline{\mathrm{H}}$ protons), 2.27 (s, $3 \mathrm{H}, \mathrm{C}_{3}$ protons at phenyl ring). MS $(\mathrm{m} / \mathrm{z}, \%): \mathbf{M}^{+}: 346.14,\left(\mathbf{M}^{+}+1: 347.18\right), \mathbf{M}^{+}-\mathrm{C}_{7} \mathrm{H}_{8}: 246.47, \mathbf{M}^{+}-$ $\mathrm{C}_{8} \mathrm{H}_{10} \mathrm{~N}_{2} \mathrm{O}: 201.22, \mathrm{M}^{+}-\mathrm{C}_{5} \mathrm{H}_{5} \mathrm{~N}: 271.12$. Anal. Calcd. (found) (\%): C, 69.35 (69.37); H, 5.24 (5.25); N, 16.17 (16.12); O, 9.24 (9.26).

N-(4-(Pyridin-2-yloxy)benzylidene)-4-(4-ethylphenyl)semicarbazide (PSSD6): Yield: $67.56 \%$, m.p. $163-165^{\circ} \mathrm{C}$, m.f. $\mathrm{C}_{21} \mathrm{H}_{20} \mathrm{~N}_{4} \mathrm{O}_{2}$; m.w. 360.41, $\mathrm{R}_{\mathrm{f}}$ 0.49. FT-IR $\left(\mathrm{KBr}, v_{\max }, \mathrm{cm}^{-1}\right)$ : 3390, 3325 (N-H str.), 3041, 2935, 2868 (C-H str.), 2000-1670 (over-tone for disubstitution on aromatic ring), $1672(\mathrm{C}=\mathrm{O}$ str.), 1648 (azomethine $\mathrm{C}=\mathrm{N}$ str.), 1592 (phenyl ring str.), 1471 (phenyl C-H in plane bending), 1453 (asym. methyl C-H bending), 1372 (sym. methyl C-H bending), 1278 (C-N str. coupled with N-H bending), 1254 (C-O str.), 1048 (N-N str.), 849 (C-H out of plane bending for disubstitution). ${ }^{1} \mathrm{H}$ NMR $\left(\mathrm{CDCl}_{3}, 300 \mathrm{MHz}\right) \delta$ in ppm: $8.26(\mathrm{~s}, 1 \mathrm{H}, \mathrm{Ph}-\mathrm{C} \underline{\mathrm{H}}=\mathrm{N}), 7.82-7.80(\mathrm{t}, 1 \mathrm{H}$, pyridine ring proton at $\left.\mathrm{C}_{4}\right), 7.78-7.76\left(\mathrm{~d}, 1 \mathrm{H}\right.$, pyridine ring proton at $\left.\mathrm{C}_{6}\right)$, 7.72-7.70 (d, $2 \mathrm{H}, m$-phenyl ring protons at $\left.\mathrm{N}_{4}\right), 7.56-7.54(\mathrm{~d}$, $2 \mathrm{H}, o$-benzyl ring protons at $\left.\mathrm{N}_{1}\right), 7.24-7.22(\mathrm{~d}, 2 \mathrm{H}, m$-benzyl ring protons at $\left.\mathrm{N}_{4}\right), 6.92-6.90(\mathrm{~d}, 2 \mathrm{H}, o$-benzyl ring protons at $\left.\mathrm{N}_{1}\right), 6.62-6.60\left(\mathrm{~d}, 1 \mathrm{H}\right.$, pyridine ring proton at $\left.\mathrm{C}_{3}\right), 6.54-6.48$ $\left(\mathrm{t}, 1 \mathrm{H}\right.$, pyridine ring proton at $\left.\mathrm{C}_{5}\right), 6.14(\mathrm{~s}, 2 \mathrm{H}$, semicarbazide $\mathrm{N} \underline{\mathrm{H}}$ protons), 2.68 (s, 3H, $\mathrm{C}_{3}$ protons at phenyl ring), 1.45 (s, $2 \mathrm{H}, \mathrm{CH}_{2}$ protons at phenyl ring). $\mathrm{MS}(\mathrm{m} / \mathrm{z}, \%): \mathrm{M}^{+}: 360.16$, $\left(\mathrm{M}^{+}+1: 361.17\right), \mathrm{M}^{+}-\mathrm{C}_{3} \mathrm{H}_{5}: 333.13, \mathrm{M}^{+}-\mathrm{C}_{8} \mathrm{H}_{10}: 258.10, \mathrm{M}^{+}-$ $\mathrm{C}_{9} \mathrm{H}_{12} \mathrm{~N}_{2} \mathrm{O}: 198.08, \mathrm{M}^{+}-\mathrm{C}_{5} \mathrm{H}_{5} \mathrm{~N}: 284.14$. Anal. Calcd. (found) (\%): C, 69.98 (69.96); H, 5.59 (5.60); N, 15.55 (15.57); O, 8.88 (8.92).

$\mathrm{N}$-(4-(Pyridin-2-yloxy)benzylidene)-4-(4-methoxyphenyl)semicarbazide (PSSD7): Yield: $62.86 \%$, m.p. 130$132{ }^{\circ} \mathrm{C}$, m.f. $\mathrm{C}_{20} \mathrm{H}_{18} \mathrm{~N}_{4} \mathrm{O}_{3}$; m.w. 362.38, $\mathrm{R}_{\mathrm{f}}$ 0.49. FT-IR ( $\mathrm{KBr}$, $\left.v_{\max }, \mathrm{cm}^{-1}\right): 3438,3318$ (N-H str.), 3025, 2976, 2881 (C-H str.), 2000-1670 (overtone for disubstitution on aromatic ring), 1666 $(\mathrm{C}=\mathrm{O}$ str.), 1647 (azomethine $\mathrm{C}=\mathrm{N}$ str.), 1587 (phenyl ring str.), 1461 (phenyl C-H in plane bending), 1452 (asym. methyl C-H bending), 1381 (sym. methyl C-H bending), 1272 (C-N str. coupled with N-H bending), 1254 (C-O str.), 1055 (N-N str.), 843 (C-H out of plane bending for disubstitution). ${ }^{1} \mathrm{H}$ NMR $\left(\mathrm{CDCl}_{3}, 300 \mathrm{MHz}\right) \delta$ in ppm: 8.31 (s, $\left.1 \mathrm{H}, \mathrm{Ph}-\mathrm{C} \underline{\mathrm{H}}=\mathrm{N}\right), 7.72-7.70$ $\left(\mathrm{d}, 2 \mathrm{H}, o\right.$-benzyl ring protons at $\left.\mathrm{N}_{1}\right), 7.68-7.64(\mathrm{t}, 1 \mathrm{H}$, pyridine ring proton at $\left.\mathrm{C}_{4}\right), 7.59-7.56(\mathrm{~d}, 2 \mathrm{H}, m$-phenyl ring protons at $\left.\mathrm{N}_{4}\right), 7.40-7.38\left(\mathrm{~d}, 1 \mathrm{H}\right.$, pyridine ring proton at $\left.\mathrm{C}_{6}\right), 7.06-7.02(\mathrm{~d}$, $2 \mathrm{H}, m$-benzyl ring protons at $\left.\mathrm{N}_{1}\right), 6.82-6.80(\mathrm{~d}, 2 \mathrm{H}, m$-phenyl ring protons at $\left.\mathrm{N}_{4}\right), 6.64-6.62(\mathrm{~d}, 1 \mathrm{H}$, pyridine ring proton at $\left.\mathrm{C}_{3}\right), 6.52-6.48\left(\mathrm{t}, 1 \mathrm{H}\right.$, pyridine ring proton at $\left.\mathrm{C}_{5}\right), 6.08(\mathrm{~s}, 2 \mathrm{H}$, semicarbazide $\mathrm{N} \underline{\mathrm{H}}$ protons), 3.81 (s, $3 \mathrm{H}, \mathrm{C}_{3}$ protons at phenyl ring). $\mathrm{MS}(\mathrm{m} / \mathrm{z}, \%): \mathrm{M}^{+}: 362.14,\left(\mathrm{M}^{+}+1: 363.13\right), \mathrm{M}^{+}-\mathrm{CH}_{4} \mathrm{O}$ : 333.18, $\mathrm{M}^{+}-\mathrm{C}_{7} \mathrm{H}_{8} \mathrm{O}: 257.10, \mathrm{M}^{+}-\mathrm{C}_{8} \mathrm{H}_{10} \mathrm{~N}_{2} \mathrm{O}: 198.15, \mathrm{M}^{+}-\mathrm{C}_{5} \mathrm{H}_{5} \mathrm{~N}$ : 286.11. Anal. Calcd. (found) (\%): C, 66.29 (66.31); H, 5.01 (5.04); N, 15.46 (15.42); O, 13.25 (13.22).

N-(4-Pyridin-2-yloxy)benzylidene)-4-(4-nitrophenyl)semicarbazide (PSSD8): Yield: $60.25 \%$, m.p. $218-220^{\circ} \mathrm{C}$, m.f. $\mathrm{C}_{19} \mathrm{H}_{15} \mathrm{~N}_{5} \mathrm{O}_{4}$; m.w. 377.35, $\mathrm{R}_{\mathrm{f}}$ 0.67. FT-IR $\left(\mathrm{KBr}, v_{\max }, \mathrm{cm}^{-1}\right)$ : 3432, 3321 (N-H str.), 3025, 2972, 2886 (C-H str.), 2000-1670 (overtone for disubstitution on aromatic ring), $1661(\mathrm{C}=\mathrm{O}$ str.), 1641 (azomethine $\mathrm{C}=\mathrm{N}$ str.), 1592 (phenyl ring str.), 1462 (phenyl $\mathrm{C}-\mathrm{H}$ in plane bending), 1445 (asym. methyl $\mathrm{C}-\mathrm{H}$ bending),
1375 (sym. methyl C-H bending), 1287 (C-N str. coupled with $\mathrm{N}-\mathrm{H}$ bending), 1247 (C-O str.), 1042 (N-N str.), 849 (C-H out of plane bending for disubstitution). ${ }^{1} \mathrm{H}$ NMR $\left(\mathrm{CDCl}_{3}, 300\right.$ $\mathrm{MHz}) \delta$ in ppm: 8.36-8.34 (d, $2 \mathrm{H}, m$-phenyl ring protons at $\left.\mathrm{N}_{4}\right), 8.22(\mathrm{~s}, 1 \mathrm{H}, \mathrm{Ph}-\mathrm{C} \underline{\mathrm{H}}=\mathrm{N}), 7.98-7.96(\mathrm{~d}, 2 \mathrm{H}, m$-phenyl ring protons at $\left.\mathrm{N}_{4}\right), 7.88-7.86\left(\mathrm{~d}, 1 \mathrm{H}\right.$, pyridine ring proton at $\left.\mathrm{C}_{4}\right)$, 7.72-7.70 (d, $1 \mathrm{H}$, pyridine ring proton at $\left.\mathrm{C}_{6}\right), 7.52-7.50(\mathrm{~d}$, $2 \mathrm{H}, o$-benzyl ring protons at $\left.\mathrm{N}_{1}\right), 7.02-7.00(\mathrm{~d}, 2 \mathrm{H}, o$-benzyl ring protons at $\left.\mathrm{N}_{1}\right), 6.92-6.90(\mathrm{~d}, 1 \mathrm{H}$, pyridine ring proton at $\left.\mathrm{C}_{3}\right), 6.42-6.40\left(\mathrm{t}, 1 \mathrm{H}\right.$, pyridine ring proton at $\left.\mathrm{C}_{5}\right), 6.08(\mathrm{~s}, 2 \mathrm{H}$, semicarbazide $\mathrm{NH}$ protons). $\mathrm{MS}(\mathrm{m} / \mathrm{z}, \%): \mathrm{M}^{+}: 377.11,\left(\mathrm{M}^{+}+1\right.$ : 378.12), $\mathrm{M}^{+}-\mathrm{HNO}_{2}: 333.14, \mathrm{M}^{+}-\mathrm{C}_{6} \mathrm{H}_{5} \mathrm{NO}_{2}: 257.10, \mathrm{M}^{+}-\mathrm{C}_{7} \mathrm{H}_{7} \mathrm{~N}_{3} \mathrm{O}_{3}$ : 199.08, $\mathrm{M}^{+}-\mathrm{C}_{5} \mathrm{H}_{5} \mathrm{~N}$ : 302.09. Anal. Calcd. (found) (\%): C, 60.47 (60.52); H, 4.01 (4.04); N, 18.56 (18.61); O, 16.96 (16.92).

Pharmacological evaluation: The newly synthesized $N$ (4-(pyridin-2-yloxy)benzylidene)-4-[4-(substituted)phenyl]semicarbazides (PSSD1-8) were subjected to anticonvulsant screening according to the protocols of anticonvulsant drug development (ADD) program of National Institute of Health, Bethesda, USA. Albino mice of either sex weighing between 25-30 $\mathrm{g}$ were used in this study. All albino mice employed in this study were approved by the Institutional Animal Ethics Committee of Sapience Bioanalytical Research Laboratory, Bhopal, India (1413/PO/E/S/11/cpcsea) and experiment was carried out as per CPCSEA guidelines. The animals were kept in large spacious hygienic cages during the course of the experimental period. The animals had free access to standard commercial diet and water ad libitum and were kept in rooms maintained at $22 \pm 1^{\circ} \mathrm{C}$ with $12 \mathrm{~h}$ light-dark cycle. The animals were divided into three groups of six animals each: Group I: Control group (distilled water treated). Group II: Test group (test compounds were dissolved in polyethylene glycol-400 and administered at a dose of 30,100,300 mg/kg i.p. doses), Group III: Standard group on reference drug (phenytoin 30 $\mathrm{mg} / \mathrm{kg}$ i.p.). Anticonvulsant activity was established by maximal electroshock (MES) and subcutaneous metrazole (scMET) models at drug doses of 30,100 and $300 \mathrm{mg} / \mathrm{kg}$ at three different time intervals. Neurotoxicity of the compound was determined by rotorod method.

Maximum electroshock (MES) seizure test: Each treatment group and vehicle control group consisted of six animals; $60 \mathrm{~Hz}$ of alternating current (50 mA in mice) was applied via ear-clip electrodes for $0.2 \mathrm{~s}$. Mice were tested at $30 \mathrm{~min}$ and $4 \mathrm{~h}$ following doses of 30,100 and $300 \mathrm{mg} / \mathrm{kg}$ of test compound. The test compounds were dissolved polyethylene glycol (PEG) and injected intraperitoneally (i.p.) at dose of 30, 100 and 300 $\mathrm{mg} / \mathrm{kg}$ for $30 \mathrm{~min}$ before seizures induction. Phenytoin was used as reference drugs. Abolition of hind limb tonic extensor component indicated the test compound's ability to inhibit MES induced seizure spread and was defined as protection [8].

Subcutaneous pentylenetetrazole (scPTZ) seizure test: Animals were divided into three groups each comprising of six animals. One group was used for studying the effect of pentylenetetrazole, the second group for control and the third group to study effect with reference to the standard. The subcutaneous dose of pentylenetetrazole $(85 \mathrm{mg} / \mathrm{kg})$ at which $95 \%$ of the animals showed convulsive reaction was determined by a dose-percent effect curve. The synthesized compounds were 
administered at the three graded doses, namely 30, 100 and $300 \mathrm{mg} / \mathrm{Kg}$, intraperitoneally. At the anticipated time, pentylenetetrazole was then administered subcutaneously in the posterior midline of mice. The absence of clonic spasm in half or more of the animals in the observed time periods indicates the compounds capacity to terminate the effect of pentylenetetrazole on seizure threshold. The results of test compound were compared with control and standard compounds [9].

Neurotoxicity screening: The activity of the drugs interfering with motor coordination was checked by the rotarod test. The mice were trained to stay on an accelerating rotarod that rotates at 6 revolutions per minute. Trained animals were given i.p. injection of the test compounds in doses of 30, 100 and $300 \mathrm{mg} / \mathrm{kg}$. The rod diameter was $3.2 \mathrm{~cm}$. Neurotoxicity indicated by the inability of animal to maintain equilibrium on the rotarod for at least $1 \mathrm{~min}$ in each of three trials. The dose, at which the animals were unable to grasp the rotarod was determined. The number of animals in each group $(\mathrm{N})=6$; solvent used polyethylene glycol; dosed with 30, 100 and 300 $\mathrm{mg} / \mathrm{kg}$ were administered i.p. The figures indicate the minimum dose whereby bioactivity was demonstrated in half or more mice. The (-) indicates an absence of activity at maximum dose administered $(300 \mathrm{mg} / \mathrm{kg})$. In neurotoxicity screen, the figure indicates the dose at which no neurotoxicity was observed and the dash (-) indicates compounds are not tested [10].

Molecular docking: The molecular docking study of designed molecules was carried out in order to assess the interaction and binding modes with target receptor/enzyme using Glide extra precision (XP) Maestro 10.1 Schrodinger, running on Windows i5 (intel core processor) operating system [Schrodinger, Version 10.1, 2016]. The 2D structure for synthesized compounds was generated and then converted to their respective 3D structures with use of Ligplot. The PDB file of X-ray crystal structure of human $\gamma$-aminobutyric acid receptor, $\mathbf{G A B} \mathbf{A}_{\mathbf{A}} \mathbf{R}$ $\beta 3$ homopentamer (PDB ID: 4COF) was downloaded from RCSB protein data bank. The protein was prepared using the protein preparation wizard and grid was generated for co-crystal ligand, benzamidine using receptor grid generation. The water residues beyond $5 \AA$ were eliminated. The protein was optimized by assigning $\mathrm{H}$-bonds and minimization at OPLS 2005 force field. The docked pose of ligands and their interactions were analyzed after the end of molecular docking. The valproic acid is used as reference for docking studies.

Determination of ADME properties: The absorption, distribution, metabolism and excretion (ADME) properties of the synthesized compounds were determined using the results of a computational study. The descriptor topological polar surface area (TPSA), surface belonging to polar atoms, was shown to be strongly correlated with passive molecular transport through membranes; therefore, it facilitates the prediction of transport properties of drugs from the intestine and blood-brain barrier (BBB) crossingbydrugs [11]. The TPSA, mi Log P, number of rotatable bonds, number of hydrogen donor and acceptor atoms, molecular volume and violations of Lipinski's rule of five [12] of each synthesized compound were calculated using the Mol inspiration online property calculation tool kit [13]. The percentage of absorption (\% ABS) was calculated using TPSA [14].
Determination of $\log P$ value: Within a congeneric series, the biological activity of the synthesized compounds depended on the lipophillic character. Drugs acting on central nervous system are potent only if they cross blood-brain barrier; therefore, potency has been correlated with optimum the lipophilicity $(\log \mathrm{P})$, which is near 2 . In this study, we attempted to identify the correlation between the anticonvulsant activities of congeners and their calculated $\log P$ values. The values of Log P were experimentally determined using octanol-phosphate buffer method [15].

\section{RESULTS AND DISCUSSION}

A new series of $N$-(4-(pyridin-2-yloxy)benzylidene)-4[4-(substituted)phenyl]semicarbazides (PSSD1-8) was synthesized in an effort to obtain the drugs with improved anticonvulsant activity. The target compounds PSSD1-8 have been synthesized by reaction of 4-(pyridinyl-2-oxy)benzaldehyde and 4-(substituted)phenylsemicarbazides. All the synthesised compounds were characterized by analytical and spectroscopic methods. The compounds were evaluated for their anticonvulsant activity in MES and scMET models. Compounds PSSD5 and PSSD2 were found to be active in MES model. The maximal electroshock test is the most widely used animal model in evaluation of antiepileptic drugs. In the MES screen, compound PSSD2 showed protection $(1 / 1,4 \mathrm{~h})$ at a dose of $300 \mathrm{mg} / \mathrm{kg}$. Compound PSSD5 showed protection $(2 / 3,2 \mathrm{~h})$ at a dose of $100 \mathrm{mg} / \mathrm{kg}$ and protection $(1 / 1,4 \mathrm{~h})$ at a dose of $300 \mathrm{mg} / \mathrm{kg}$. None of the compounds showed protection in scMET test, a test used to identify compounds that elevate seizure threshold. Minor toxicity $(1 / 2,4 \mathrm{~h})$ as motor impairment was observed at a dose of $300 \mathrm{mg} / \mathrm{kg}$ with compound PSSD4. None of the other compounds showed neurotoxicity in the highest administered dose $(300 \mathrm{mg} / \mathrm{kg})$. The present study revealed that compounds PSSD5 and PSSD2 attenuated MES induced tonic seizures indicating anticonvulsant activity. The MES test activity of compounds showed their potential to prevent seizure spread and identified agents with profound activity against generalized tonic clonic seizures (Table-1).

The molecular docking study of the synthesized molecules was carried out in order to assess the interaction and binding modes with target receptor/enzyme. All the compounds were found to strongly bind by completely occupying the active sites (at the site of benzamine, co-crystal ligand of the protein as predicted by SiteMap) in the target protein $\left(\mathrm{GABA}_{\mathrm{A}} \mathrm{R}-\beta_{3}\right)$. Only one compound found to have less docking score compared to valproic acid, the standard anti-convulsant drug and others outscored it. Almost all the compounds showed high docking scores than valproic acid, the standard anti-convulsant drug (Table-2).

Among all the titled compounds, PSSD7 was found to be most potent and have high docking score of (-5.43) (Table-2). The binding mode of compound PSSD7 assumes favourable orientation due to two hydrogen bonds compared with reference valproic acid (-4.03 as Dock Score). The docked pose (Fig. 2) of compound PSSD7 showed the hydrogen bond interaction by ALAB:201 of protein with $\mathrm{N}$ of $\mathrm{C}=\mathrm{N}$ and GLUB:155 with $\mathrm{NH}$ of pyrimidine ring in the compound along with two $\pi-\pi$ staking of pyrimidine ring with TYRA: 62 and TYRB: 157, a 


\begin{tabular}{|c|c|c|c|c|c|c|c|c|}
\hline \multirow{4}{*}{ Compounds } & \multicolumn{8}{|c|}{$\begin{array}{c}\text { TABLE-1 } \\
\text { ANTICONVULSANT ACTIVITY AND NEUROTOXICITY OF } \\
\text { RIDIN-2-YLOXY)BENZYLIDENE)-4-[4-(SUBSTITUTED)PHENYL]SEMICARBAZIDES (PSSD 1-8) }\end{array}$} \\
\hline & \multicolumn{8}{|c|}{ Intraperitoneal injection in rat ${ }^{\mathrm{a}}$} \\
\hline & \multicolumn{3}{|c|}{ MES screen (h) } & \multicolumn{3}{|c|}{ scMET screen $(\mathrm{h})$} & \multicolumn{2}{|c|}{ Neurotoxicity screen (h) } \\
\hline & 0.5 & 2.0 & 4.0 & 0.5 & 2.0 & 4.0 & 0.5 & 4.0 \\
\hline PSSD1 & - & - & - & - & - & - & - & - \\
\hline PSSD2 & - & - & $300^{\mathrm{b}}$ & - & - & - & - & - \\
\hline PSSD3 & - & - & - & - & - & - & - & - \\
\hline PSSD4 & - & - & - & - & - & - & - & $300^{c}$ \\
\hline PSSD5 & - & $100^{\mathrm{b}}$ & $300^{\mathrm{b}}$ & - & - & - & - & - \\
\hline PSSD6 & - & - & - & - & - & - & - & - \\
\hline PSSD7 & - & - & - & - & - & - & - & - \\
\hline PSSD8 & - & - & - & - & - & - & - & - \\
\hline Phenytoin & 30 & 30 & 30 & - & - & - & 100 & 100 \\
\hline Normal saline & - & - & - & - & - & - & - & - \\
\hline
\end{tabular}

${ }^{\text {a }}$ Doses of 30,100 and $300 \mathrm{mg} / \mathrm{kg}$ were administered. The figures in the table indicate the minimum dose whereby bioactivity was demonstrated in half or more of the rat. The dash (-) indicates an absence of activity at maximum dose administered $(300 \mathrm{mg} / \mathrm{kg})$.

TABLE-2

DOCKING SCORES OF PSSD 1-8 AND VALPROIC ACID

\begin{tabular}{|c|c|c|c|c|c|c|c|c|c|c|}
\hline Ligand & GScore & $\begin{array}{l}\text { Dock } \\
\text { score }\end{array}$ & $\begin{array}{c}\text { Lipophilic } \\
\text { EvdW }\end{array}$ & $\begin{array}{c}\text { Phob } \\
\text { En }\end{array}$ & $\begin{array}{l}\text { Phob } \\
\text { EnHB }\end{array}$ & H-Bond & Electro & PiCat & $\begin{array}{l}\text { Expos } \\
\text { penal }\end{array}$ & Rot penal \\
\hline PSSD7 & -5.43 & -5.43 & -2.87 & -1.52 & -0.75 & -0.01 & -0.40 & 0 & 0.48 & 0.44 \\
\hline PSSD8 & -5.25 & -5.25 & -2.48 & -1.55 & -0.75 & -0.42 & -0.61 & 0 & 0.54 & 0.41 \\
\hline PSSD4 & -4.86 & -4.86 & -3.03 & -1.65 & -0.75 & 0.00 & -0.30 & 0 & 1.22 & 0.40 \\
\hline PSSD3 & -4.83 & -4.83 & -3.12 & -1.01 & 0.00 & -0.60 & -0.31 & 0 & 0.44 & 0.30 \\
\hline PSSD2 & -4.80 & -4.80 & -2.89 & -1.67 & -0.75 & -0.48 & -0.49 & 0 & 0.90 & 0.37 \\
\hline PSSD1 & -4.43 & -4.43 & -2.86 & -1.48 & 0.00 & 0.00 & 0.00 & 0 & 0.41 & 0.44 \\
\hline PSSD5 & -4.13 & -4.13 & -3.05 & -0.97 & 0.00 & -0.54 & -0.48 & 0 & 0.18 & 0.48 \\
\hline PSSD6 & -2.60 & -2.60 & -3.08 & -0.60 & 0.00 & -0.08 & -0.24 & 0 & 1.25 & 0.44 \\
\hline Valproic acid & -4.03 & -4.03 & -1.29 & -1.40 & -1.00 & 0.00 & -0.35 & 0 & 0.00 & 0.60 \\
\hline
\end{tabular}

*Note: Results are obtained in XP visualizer (application which shows results obtained by Extra precision docking) and the short forms used stands for G-score: Glide Sore; PhobEnHB: Reward for hydrophobically packed H-bond; PhobEn: Hydrophobic enclosure reward; Penalties: Polar atom burial and desolvation penalties, and penalty for intra-ligand contacts; ExposPenal: Penalty for solvent-exposed ligand groups; cancels van der Waals terms; RotPenal: Rotatable bond penalty.
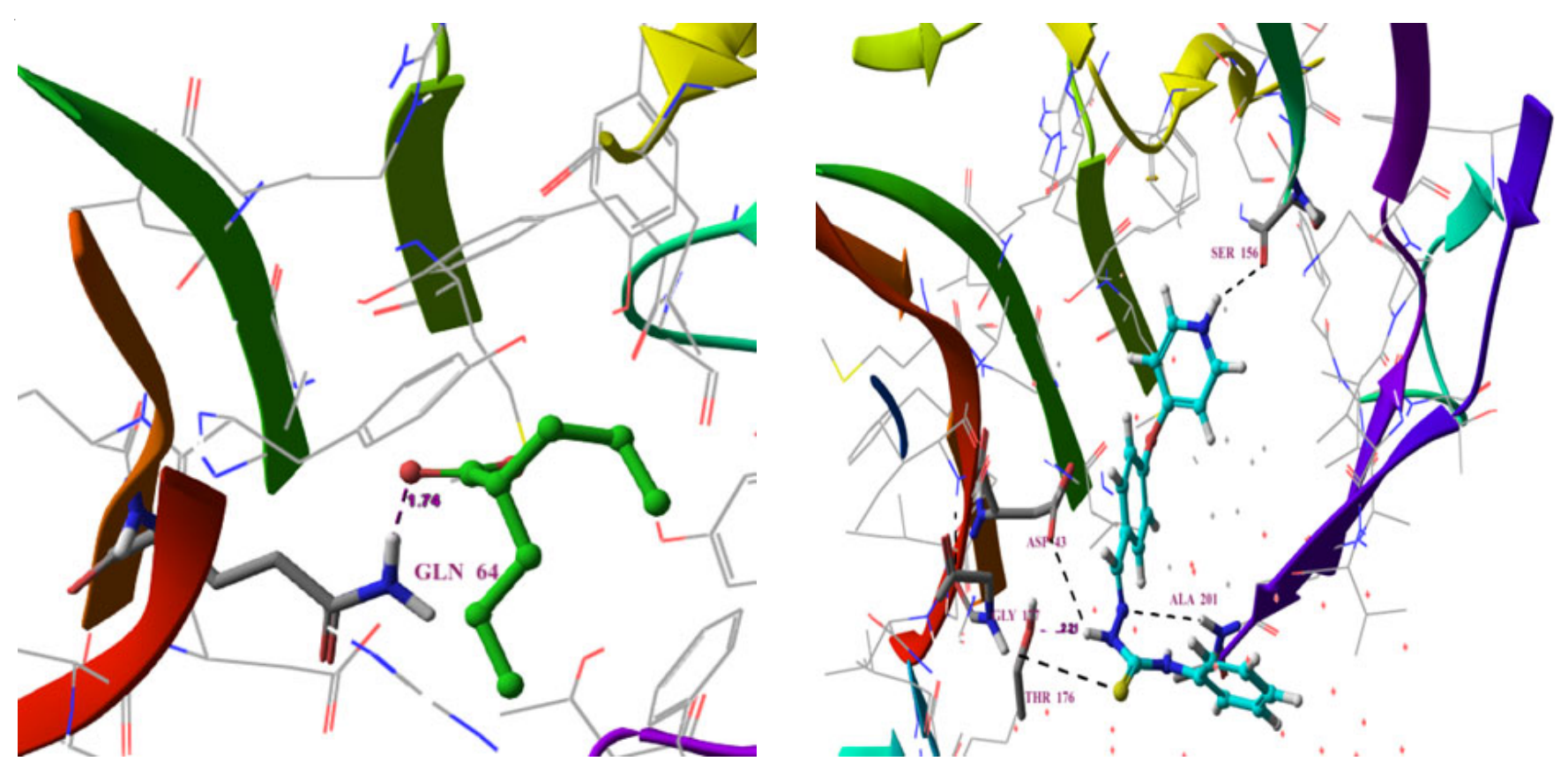

Fig. 2. 3D Docked pose of PSSD7 (left) and valproic acid (right)

salt bridge of NH with GLUB: 155 and negative charges formed with TYRB: 205,157 of the same ring and that of valproic acid in which one carbonyl group forms H-bonds with GLYA 64. Our lead enjoys good hydrophobic enthalpy reward (-2.87) 


\begin{tabular}{|c|c|c|c|c|c|c|c|c|c|}
\hline \multicolumn{10}{|c|}{$\begin{array}{c}\text { TABLE-3 } \\
\text { PHARMACOKINETIC PARAMETERS IMPORTANT FOR GOOD ORAL BIOAVAILABILITY OF } \\
N \text {-(4-(PYRIDIN-2-YLOXY) BENZYLIDENE)-4-[4-(SUBSTITUTED)PHENYL]SEMICARBAZIDES (PSSD1-8) }\end{array}$} \\
\hline Compounds & $\mathrm{R}$ & $\%$ ABS & $\operatorname{TPSA}\left(\mathrm{A}^{2}\right)$ & n-ROTB & MW & MV & $\begin{array}{c}\mathrm{n}-\mathrm{OHNH} \\
\text { donors }\end{array}$ & $\begin{array}{c}\mathrm{n} \text {-ON } \\
\text { acceptors }\end{array}$ & $\begin{array}{l}\text { Lipinski's } \\
\text { violations }\end{array}$ \\
\hline Rule & - & - & - & - & $<500$ & - & $<5$ & $<10$ & $\leq 1$ \\
\hline PSSD1 & $\mathrm{H}$ & 82.9 & 75.61 & 5 & 232.36 & 298.75 & 2 & 6 & 0 \\
\hline PSSD2 & $\mathrm{Cl}$ & 82.9 & 75.61 & 5 & 366.81 & 312.27 & 2 & 6 & 0 \\
\hline PSSD3 & $\mathrm{Br}$ & 82.9 & 75.61 & 5 & 411.26 & 316.62 & 2 & 6 & 1 \\
\hline PSSD4 & F & 82.9 & 75.61 & 5 & 350.35 & 303.67 & 2 & 6 & 0 \\
\hline PSSD5 & $\mathrm{CH}_{3}$ & 82.9 & 75.61 & 5 & 346.39 & 315.30 & 2 & 6 & 0 \\
\hline PSSD6 & $\mathrm{C}_{2} \mathrm{H}_{5}$ & 82.9 & 75.61 & 6 & 360.42 & 298.75 & 2 & 6 & 1 \\
\hline PSSD7 & $\mathrm{OCH}_{3}$ & 79.7 & 84.85 & 6 & 362.39 & 324.28 & 2 & 7 & 0 \\
\hline PSSD8 & $\mathrm{NO}_{2}$ & 67.1 & 121.44 & 5 & 377.36 & 322.07 & 2 & 9 & 0 \\
\hline
\end{tabular}

compared to standard (-1.29) over other compounds and is having very less rotatable bond penalty of 0.44 whereas the standard compound valproic acid suffers with high rotatable bond penalty 0.60 . Whereas in-case of surface exposed penalty, it suffers a little bit (0.48) which is of less importance in front of rotatable bond penalty as shown in (Fig. 3). High rewards and less penalties of ligand PSSD7 is the rationale for high docking score and potency towards the receptor. The docked pose of second most dock ranked PSSD8 with d-score -5.25 has no surface penalty but rotatable bond penalty (0.41) which is comparable to the best one and slightly less hydrophobic enthalpy reward -2.48 possessing good hydrogen bond interactions with receptor [15].

A computational study for determination of ADME properties of the synthesized compounds was performed. All titled compounds exhibited a good \% ABS ranging from 67.1 to $82.9 \%$ (Table-3). Except for compounds PSSD3 and PSSD6, none other compound violated Lipinski's parameters, making them potentially promising agents for treatment of epilepsy. It was attempted to correlate the anticonvulsant activity of congeners with their calculated Log P value (Table-4). All compounds showed lipophilic character. As observed some of the experimental values were in good agreement with the theoretical values suggesting that these have an excellent potential for CNS activity.

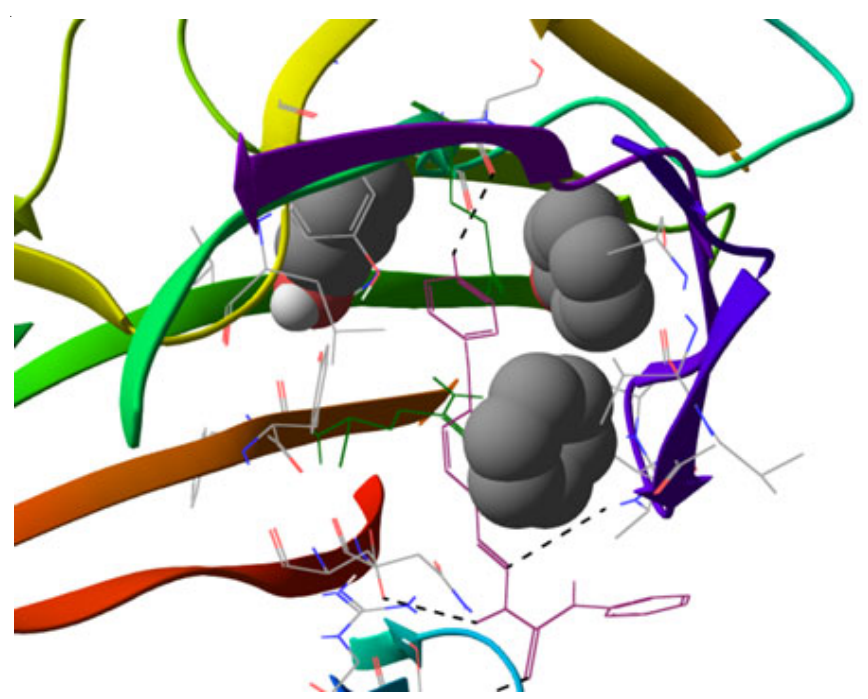

\section{TABLE-4}

LOG P VALUE FOR N-(4-(PYRIDIN-2-YLOXY)BENZYLIDENE)4-[4-(SUBSTITUTED)PHENYL]SEMICARBAZIDES (PSSD1-8)

\begin{tabular}{ccc}
\hline Compounds & Experimental Log P & $\begin{array}{c}\text { Experimental Log P } \\
(\text { miLog Pa })\end{array}$ \\
\hline Rule & - & $\leq 5$ \\
PSSD1 & 4.02 & 4.27 \\
PSSD2 & 4.67 & 4.95 \\
PSSD3 & 4.88 & 4.95 \\
PSSD4 & 4.26 & 4.43 \\
PSSD5 & 4.56 & 4.72 \\
PSSD6 & 4.92 & 4.97 \\
PSSD7 & 3.98 & 4.32 \\
PSSD8 & 3.87 & 4.23 \\
\hline
\end{tabular}

\section{Conclusion}

The above study concluded that the compounds $N$-(4-(pyridi2-yloxy)benzylidene)-4-(4-chlorophenyl)semicarbazide (PSSD2) and $N$-(4-(pyridin-2-yloxy)benzylidene)-4- $p$-tolylsemicarbazide (PSSD5) showed anticonvulsant activity in MES model and thus have the potential to prevent seizure spread. $N$-(4-(pyridin-2-yloxy)benzylidene)-4-[4-(substituted)phenyl]semicarbazides (PSSD1-8) showed good docking with human $\gamma$-aminobutyric acid receptor $\left(\mathrm{GABA}_{\mathrm{A}} \mathrm{R}-\beta 3\right)$ and the total docking score is found to be a comparable to reference drug valproic

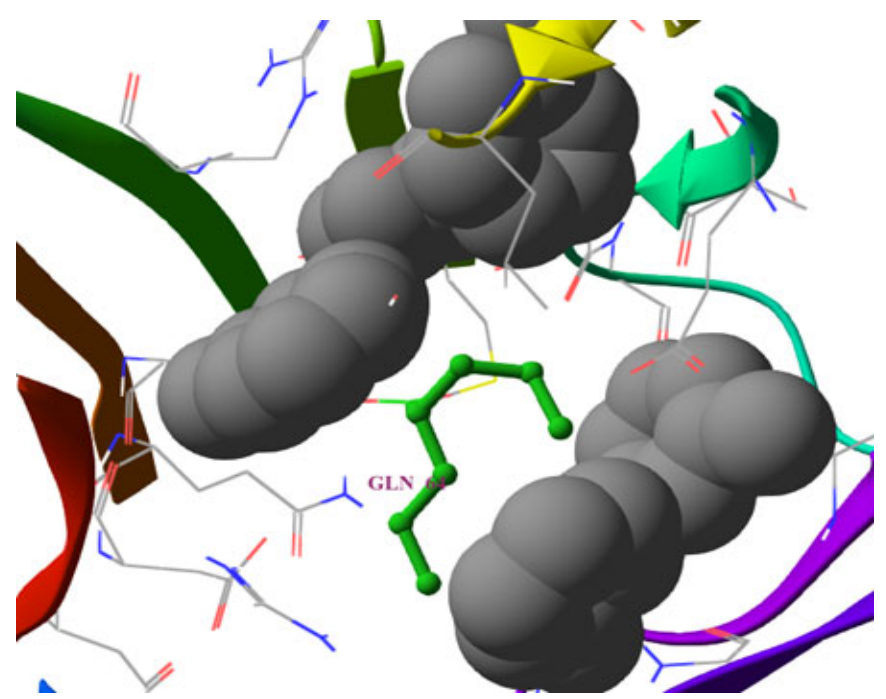

Fig. 3. 3D pose (in left) of PSSD7 (represented in green) enjoying enthalpy reward (portion of the ligand denoted in ball \& socket form). At right, less but completely, the standard VPA is rewarded 
acid. This shows that the probable mechanism of action of these compounds may be augmentation of GABAergic activity in the brain.

\section{ACKNOWLEDGEMENTS}

The authors gratefully acknowledge Prof. Ashish Singhai, Director \& HOD, Department of Natural Chemistry, Sapience Bioanalytical Research Lab, Bhopal, India; Prof. M. Shaharyar, Department of Pharmaceutical Chemistry, Jamia Hamdard, New Delhi, India and Prof. Dr. A.K. Ghosh, Vice-Chancellor, IFTM University, Moradabad, India for providing help and support during the research work.

\section{CONFLICT OF INTEREST}

The authors declare that there is no conflict of interests regarding the publication of this article.

\section{REFERENCES}

1. R.G. Greenberg, C. Melloni, H. Wu, D. Gonzalez, L. Ku, K.D. Hill, C.P. Hornik, N. Zheng, W. Jiang, M. Cohen-Wolkowiez and J.T. Guptill, Clin. Neuropharmacol., 39, 232 (2016); https://doi.org/10.1097/WNF.0000000000000172.

2. M.J. Ahsan, Cent. Nerv. Syst. Agents Med. Chem., 13, 148 (2013); https://doi.org/10.2174/18715249113136660016.

3. D.K. Jain, A. Singh, V.K. Patel, R. Veerasamy, N. Aggarwal and H. Rajak, Cent. Nerv. Syst. Agents Med. Chem., 17, 30 (2017); https://doi.org/10.2174/1871524916666160411143941.
4. S.N. Pandeya, A.A. Khan and A. Srivastava, J. Chem. Pharm. Res., 3, 456 (2011).

5. L. Tripathi, R. Singh and J.P. Stables, Eur. J. Med. Chem., 46, 509 (2011); https://doi.org/10.1016/j.ejmech.2010.11.030.

6. A. Jain, S. Patil and A. Gajbhiye, Int. J. Pharmacol. Pharm. Technol., 1, 2277 (2015).

7. L. Tripathi, P. Kumar, R. Singh and J.P. Stables, Eur. J. Med. Chem., 47, 153 (2012); https://doi.org/10.1016/j.ejmech.2011.10.038.

8. R.L. Krall, J.K. Penry, B.G. White, H.J. Kupferberg and E.A. Swinyard, Epilepsia, 19, 409 (1978); https://doi.org/10.1111/j.1528-1157.1978.tb04507.x.

9. E.A. Swinyard, J.H. Woodhead, H.S. White and M.R. Franklin, Antiepileptic Drugs, Raven-Press, New York, USA, vol. III, pp. 989-995 (1989).

10. P. Yogeeswari, D. Sriram, V. Saraswat, J.V. Ragavendran, M.M. Kumar, S. Murugesan, R. Thirumurugan and J.P. Stables, Eur. J. Pharm. Sci., 20, 341 (2003); https://doi.org/10.1016/j.ejps.2003.08.002.

11. P. Ertl, B. Rohde and P. Selzer, J. Med. Chem., 43, 3714 (2000); https://doi.org/10.1021/jm000942e.

12. C.A. Lipinski, L. Lombardo, B.W. Dominy and P.J. Feeney, Adv. Drug Deliv. Rev., 46, 3 (2001); https://doi.org/10.1016/S0169-409X(00)00129-0.

13. Molinspiration Cheminformatics, Bratislava, Slovak Republic. http://www.molinspiration.com/servies/properties.html [accessed 16.08.2017].

14. Y.H. Zhao, M.H. Abraham, J. Le, A. Hersey, C.N. Luscombe, G. Beck, B. Sherborne and I. Cooper, Pharm. Res., 19, 1446 (2002); https://doi.org/10.1023/A:1020444330011.

15. V.A. Farrar, M. Ciechanowicz-Rutkowska, J. Grochowski, P. Serda, T. Pilati, G. Filippini, C.N. Hinko, A. El-Assadi and J.A. Moore, J. Med. Chem., 36, 3517 (1993); https://doi.org/10.1021/jm00075a005. 